\title{
Real-time active noise control with preservation of desired sound
}

DOI:

10.1016/j.apacoust.2019.07.019

\section{Document Version}

Submitted manuscript

Link to publication record in Manchester Research Explorer

\section{Citation for published version (APA):}

Zhou, C., \& Utyuzhnikov, S. (2020). Real-time active noise control with preservation of desired sound. Applied Acoustics, 157, 1. [106971]. https://doi.org/10.1016/j.apacoust.2019.07.019

\section{Published in:}

Applied Acoustics

\section{Citing this paper}

Please note that where the full-text provided on Manchester Research Explorer is the Author Accepted Manuscript or Proof version this may differ from the final Published version. If citing, it is advised that you check and use the publisher's definitive version.

\section{General rights}

Copyright and moral rights for the publications made accessible in the Research Explorer are retained by the authors and/or other copyright owners and it is a condition of accessing publications that users recognise and abide by the legal requirements associated with these rights.

\section{Takedown policy}

If you believe that this document breaches copyright please refer to the University of Manchester's Takedown Procedures [http://man.ac.uk/04Y6Bo] or contact uml.scholarlycommunications@manchester.ac.uk providing relevant details, so we can investigate your claim.

\section{OPEN ACCESS}




\title{
Real-time active noise control with preservation of desired sound
}

\author{
C. Zhou ${ }^{1}$, S. Utyuzhnikov ${ }^{1,2}$ \\ ${ }^{1}$ University of Manchester, Manchester, M13 9PL, UK, \\ s.utyuzhnikov@manchester.ac.uk, \\ ${ }^{2}$ Moscow Institute of Physics \& Technology, Russia
}

\begin{abstract}
In the active sound control problem a quite arbitrary bounded domain is shielded from noise generated outside by implementing secondary sound sources on the perimeter. The sound generated by interior sources, also known as desired sound, is supposed to be protected inside the shielded domain. If the desired sound is present, it is required to remain it unaffected by the control. This problem becomes much more complicated since the secondary sources have a reverse effect on the input data. In the current paper, a novel potential-based algorithm for active sound control is applied to attenuate noise with the preservation of desired sound. The uniqueness of this algorithm is that it realizes an active sound control via the utilization of surface potentials which have a projection property. The algorithm automatically removes the contribution of controls and desired sound from the total input field to be measured. The applicability of the algorithm is demonstrated via a series of numerical experiments. In addition, some factors such as the effect of the number of controls and sensors are also discussed.
\end{abstract}

Key words: active sound control; noise attenuation; nonlocal control; potentials; 
projectors; desired sound.

\section{Introduction}

The active sound control (ASC) has proved to be an efficient method to attenuate noise. With public awareness about the environmental noise hazard rising, demands increase towards intensive research on noise control, which results in rapid development of ASC in the last fifty years. In the ACS problems, it is usually assumed that a bounded domain is shielded from the noise generated outside. The noise attenuation is realized by implementing secondary sound sources, also known as controls, on the perimeter of a domain to be shielded. The Huygens principle is the basic foundation of the ASC. Based on the Huygens principle, anti-noise can be generated by controls situated on a boundary surface around the protected domain. In practical applications, the ASC is well suited for low frequency noise but not efficient for the attenuation of high-frequencies due to the space and time limitations on the control system. In contrast to ASC, the passive control exploiting some purely mechanical manners is mostly applicable for high frequency noise attenuation. These two approaches are often combined to shield an area from a broadband noise.

The existence of desired sound is usually not presumed in the ASC problem [1]. The preservation of an interior component makes the ACS problem much more complicated since it should ideally leave the desired field unaffected by the controls. Malyuzhinets [2] was the first who analyzed the ASC problem for the case when the interior component is present. In that paper the problem formulation was based on the Helmholtz equation under the assumption of 
knowledge of total acoustic field from only the primary sources.

A potential-based approach was developed to attenuate noise with the preservation of desired sound in a number of publications. Different problem formulations were considered for a time-harmonic acoustic field in $[3,4,6 ?]$. Afterwards, the method was extended to analyze the ASC problem in composite domains (see, e.g., [7-9] ). For the first time, the nonlinear potentials were implemented to solve a nonlinear statement of the problem in [10]. An unsteady formulation of the problem was considered in [11]. A number of physical and numerical experiments were conducted to implement the potential-based ASC approach in [12-16].

In all these papers mentioned above, the total field from the primary sources is supposed to be available nearby the perimeter of the the protected region. This leads to a local control since the operation of each control is independent. However, practically it is impossible to measure the primary field since in real time the total field is affected by the controls via a reverse effect [17]. This happens because the desired acoustic sound is doubled outside the protected domain [18]. Thus, the controls can affect the input data. There have been some attempts to overcome this problem with the use of different techniques such as directional measurements and surface integral control [19-21]. Based on these techniques, a different level of success was achieved [22-24].

A novel method is proposed to cope with the reverse effect of controls in [25-27]. The input data are supposed to be measured on the external side of a boundary surface around the shielded domain. As such, the input data contain not only the primary field but also the secondary field. The controls are generated via the calculation of the Calderon surface potentials which 
are projectors. Therefore, the controls become non-local since their operation depends on the total acoustic field over the entire closed surface. As a result, the algorithm can effectively remove the contribution of the interior sound including secondary field from the entire input field. Thus, the potential-based ASC method is capable of automatically distinguishing the contribution of noise from the total acoustic field at the perimeter.

In the current paper, we carry out numerical experiments of the ASC based on the algorithm proposed in [25-27] to study its properties. The paper is organized as follows. A mathematical formulation of the ASC problem is introduced in the next section. The implementation of the potential-based algorithm is described in Section 3. Finally, numerical results are presented and discussed in Section 4 which is followed by the Conclusion.

\section{General statement of the ASC problem}

Let us consider some domain $D_{0}: D_{0} \subseteq R^{3}$, a shielded subdomain $D^{+}: D^{+} \subset$ $D_{0}$ with boundary $\Gamma$ and domain $D^{-}:=D_{0} \backslash \overline{D^{+}}$where noise sources are situated.

Next, consider the problem in the frequency domain. Assume the acoustic field in domain $D_{0}$ is described by the following linear boundary value problem (BVP) :

$$
\begin{aligned}
& \triangle p+k^{2} p=f, \\
& p \in \Xi_{D_{0}},
\end{aligned}
$$

where $p$ is the total acoustic field; $k$ is the wave number; $f$ corresponds to the 


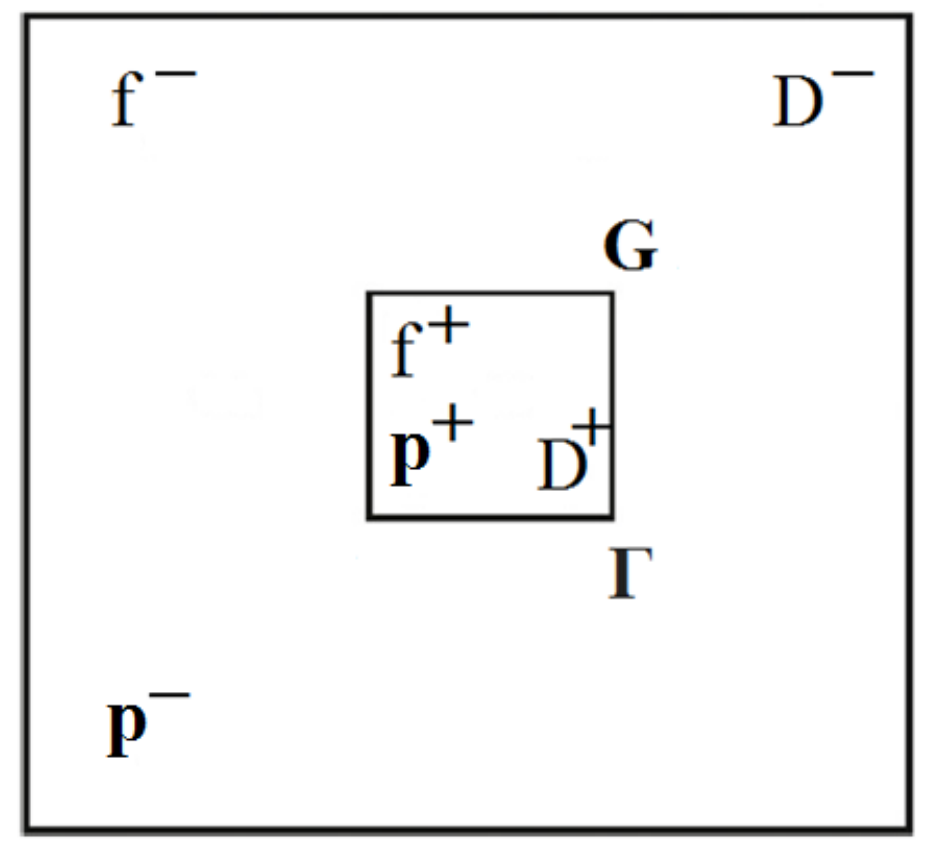

Fig. 1. Domain sketch

acoustic sources; $\Xi_{D_{0}}$ is a functional space such that the solution of BVP (1) and (2) is unique.

The sound sources $f$ consist of noise sources $f^{-}$and desired sound sources $f^{+}$:

$$
\begin{aligned}
& f=f^{+}+f^{-}, \\
& \operatorname{supp} f^{+} \subset D^{+}, \\
& \operatorname{supp} f^{-} \subset D^{-} .
\end{aligned}
$$

In turn, the total acoustic field $p$ consists of desired sound $p^{+}$and noise $p^{-}$:

$$
p=p^{+}+p^{-} .
$$

In the problem, noise $p^{-}$is attenuated inside the protected domain $D^{+}$while the desired field $p^{+}$is preserved. The active control presumes the implemen- 
tation of secondary sound sources $G$ on boundary surface $\Gamma$. Then, the formulation of the problem is reduced to the following inverse source problem:

$$
\begin{aligned}
& \triangle p_{c}+k^{2} p_{c}=f+G \\
& \operatorname{supp} G \subset D_{0} \backslash D^{+}, \\
& G \in \Xi_{D_{0}} .
\end{aligned}
$$

It is required to find $G$ such that in $D^{+}: p_{c}=p^{+}$.

\section{Implementation of ASC algorithm}

\subsection{Local control}

First, consider a local control. Assume that the total primary field $p_{\Gamma}$ is known at the perimeter $\Gamma$. Then, control $G$ is given (see e.g. [11], [25]) :

$$
G(\Gamma)=\delta(\Gamma) \frac{\partial p_{\Gamma}}{\partial \mathbf{n}}+p_{\Gamma} \frac{\partial \delta(\Gamma)}{\partial \mathbf{n}}
$$

where $\mathbf{n}$ corresponds to the outward unit normal to the boundary surface $\Gamma$; $\delta(\Gamma)$ is the surface delta-function associated with the boundary surface $\Gamma$.

In this case, the control is local since the action of the control at each point of $\Gamma$ is independent. Then, it generates anti-noise $-p^{-}$to attenuate noise $p^{-}$. However, the control generates not only anti-noise $-p^{-}$inside the protected domain $D^{+}$but also a field outside, which is equal to desired sound $p^{+}$[11]. Therefore, it doubles the desired sound outside the protected region.

Thus, if the control operates, then the primary field $p_{\Gamma}$ becomes unavailable from measurements. In order to overcome this reverse effect, a nonlocal 
potential-based ASC method is introduced in the next section.

\subsection{Nonlocal control}

In the potential-based algorithm we can take into account the reverse effect of controls in the ASC. According to [25], suppose that the input data $p_{c \Gamma_{0}}$ is measured on a surface $\Gamma_{0}$ situated in $D^{-}$(see Figure 2). In contrast to the approach described in the previous section, we consider the total field $p_{c \Gamma_{0}}$ from the primary and secondary sources available at the external surface $\Gamma_{0}$. Then, we obtain:

$$
\begin{gathered}
p_{e \mid \Gamma}^{-}(\mathbf{x})=\int_{\Gamma_{0}}\left(G r(\mathbf{x} \mid \mathbf{y}) \frac{\partial p_{c \mid \Gamma_{0}}(\mathbf{y})}{\partial \mathbf{n}_{0}}-\frac{\partial G r(\mathbf{x} \mid \mathbf{y})}{\partial \mathbf{n}_{0}} p_{c \mid \Gamma_{0}}(\mathbf{y})\right) d \sigma \\
\mathbf{x} \in \Gamma, \mathbf{y} \in \Gamma_{0}
\end{gathered}
$$

where $\mathbf{n}_{0}$ corresponds to the outward unit normal to the external surface $\Gamma_{0} ; G r$ is Green's function; $\mathrm{x}$ is an observation point. Due to the projection property of the potential, $p_{e}^{-}=p^{-}$.

Then, the local control (6) described in the previous section can be realized on $\Gamma$ to attenuate noise. It is to be noted control $(6),(7)$ is non-local since its operation depends on the acoustic field over the entire closed surface $\Gamma_{0}$. As can be seen, noise can be automatically extracted from the field $p_{c \mid \Gamma_{0}}$ at the external surface $\Gamma_{0}$. In other words, this overcomes the reverse effect of controls since the contribution of desired sound and controls is removed from the total field.

Following to [25], the nonlocal control (6), (7) can be approximated by a set 


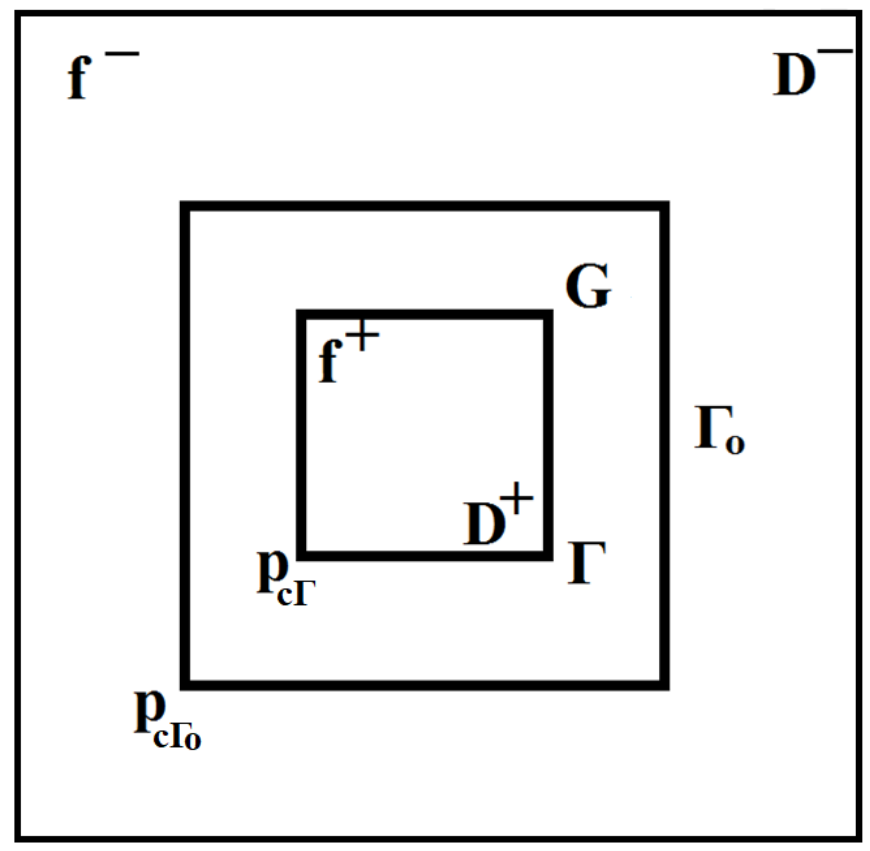

Fig. 2. Sketch of nonlocal control

of discrete sensors and controls.

Consider a discrete distribution of the sensors on $\Gamma_{0}$. For that we split the entire surface $\Gamma_{0}$ onto nonintersecting elementary segments $\sigma_{i}^{-} \quad\left(i=1, \ldots, N^{-}\right)$ with appropriate areas $\Delta \sigma_{i}^{-}$. Assume that $l_{i}=\sqrt{\Delta \sigma_{i}^{-}} \ll \mathbf{r}_{i}=\left|\mathbf{y}-\mathbf{y}_{i}\right| \quad(i=$ $\left.1, \ldots, N^{-}\right)$. Then, the first integral in (7) is approximated by a set with pressure derivatives or particle velocities:

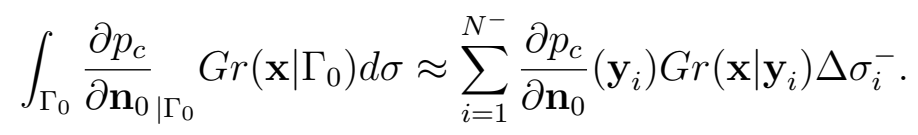

In turn, the second integral in (7) is represented by a set with sound pressures:

$$
\int_{\Gamma_{0}} \frac{\partial G r\left(\mathbf{x} \mid \Gamma_{0}\right)}{\partial \mathbf{n}_{0}}{ }_{\mid \Gamma_{0}} p_{c}\left(\Gamma_{0}\right) d \sigma \approx \sum_{i=1}^{N^{-}} \frac{\partial G r\left(\mathbf{x} \mid \mathbf{y}_{i}\right)}{\partial \mathbf{n}_{0}} p_{c}\left(\mathbf{y}_{i}\right) \Delta \sigma_{i}^{-} .
$$

Next, introduce also a discrete distribution of controls on $\Gamma$ covering the entire surface $\Gamma$ by nonintersecting elementary segments $\sigma_{i} \quad(i=1, \ldots, N)$ with appropriate areas $\Delta \sigma_{i}$. 
Approximation of the right-hand side in (7) by (8) and (9) leads to the input field on $\Gamma$ for the controls:

$$
p_{c}^{-}\left(\mathbf{x}_{i}\right)=\sum_{j=1}^{N^{-}} \Delta \sigma_{j}^{-}\left(\frac{\partial p_{c}}{\partial \mathbf{n}_{0}}\left(\mathbf{y}_{j}\right) G\left(\mathbf{x}_{i} \mid \mathbf{y}_{j}\right)-p_{c}\left(\mathbf{x}_{j}\right) \frac{\partial G}{\partial \mathbf{n}_{0}}\left(\mathbf{x}_{i} \mid \mathbf{y}_{j}\right)\right)
$$

where $\mathbf{x}_{i} \in \Delta \sigma_{i} \quad(i=1, \ldots, N), \mathbf{y}_{j} \in \Delta \sigma_{j}^{-}\left(j=1, \ldots, N^{-}\right)$.

In turn, the control function $G$ on $\Gamma$ is approximated by a set of monopoles and dipoles:

$$
\begin{aligned}
& G(\Gamma)=\frac{\partial p_{e}^{-}}{\partial \mathbf{n}} \delta(\Gamma)+\frac{\partial p_{e}^{-} \delta(\Gamma)}{\partial \mathbf{n}} \approx \\
& \sum_{i=1}^{N}\left[\frac{\partial p_{c}^{-}}{\partial \mathbf{n}}\left(\mathbf{x}_{i}\right) \delta\left(\mathbf{x}-\mathbf{x}_{i}\right)+p_{c}^{-}\left(\mathbf{x}_{i}\right) \frac{\partial \delta}{\partial \mathbf{n}}\left(\mathbf{x}-\mathbf{x}_{i}\right)\right] \Delta \sigma_{i},
\end{aligned}
$$

where $\mathbf{x}_{i} \in \Delta \sigma_{i} \quad(i=1, \ldots, N), \mathbf{n}$ is the outward normal to $\Gamma$.

Then, the nonlocal control is represented by:

$$
\begin{aligned}
& G_{0}=\sum_{i=1}^{N} \Delta \sigma_{i} * \\
& \sum_{j=1}^{N^{-}}\left[\left(A_{i j} \frac{\partial p_{c}}{\partial \mathbf{n}_{0}}+B_{i j} p_{c}\right)_{\mid \mathbf{y}_{j}} \delta\left(\mathbf{x}-\mathbf{x}_{i}\right)+\left(C_{i j} \frac{\partial p_{c}}{\partial \mathbf{n}_{0}}+D_{i j} p_{c}\right)_{\mid \mathbf{y}_{j}} \frac{\partial \delta}{\partial \mathbf{n}_{0}}\left(\mathbf{x}-\mathbf{x}_{i}\right)\right]
\end{aligned}
$$

Here, transfer matrices $A, B, C$ and $D$, which have dimensions $\left[N \times N^{-}\right]$, depend only on the coordinates of the sensors and speakers, properties of the surrounding medium and the geometry of domains bounded by $G$ and $G_{0}$. With the knowledge of Green's function, control (11) is fully determined by the geometry of the problem. Otherwise, they can be measured or calculated in advance. In the latter case, the method of difference potentials [3] along with the boundary element method [28-30] can effectively be used. 


\section{Results of ASC simulation}

In this section, the results of numerical simulation are presented. The setup of numerical simulation is composed of virtual primary sources, sensors and secondary sources. The shielded domain $D^{+}$is a cube of length equal to 1 $m$ which center coincides with the origin of the Cartesian coordinate system. First, we consider point primary sources which are presented by monopoles. An undesired sound source is located outside the protected domain at point with coordinate $(1.5 \mathrm{~m}, 1.5 \mathrm{~m}, 0 \mathrm{~m})$. A desired sound source is situated at the center of the protected domain. In addition, assume that external surface $\Gamma_{0}$ forms a cube with the sides parallel to those of cube $D^{+}$with the same center. Finally, we consider the case of a line noise source.

In the simulations, the average attenuation is calculated at $N$ observation points which are uniformly distributed in the protected region $D^{+}$:

$$
A_{s}=\frac{1}{N} \sum_{k=1}^{N} 20 \log _{10}\left|\frac{p^{-}}{\bar{p}_{c}^{-}}\right|
$$

where $\bar{p}_{c}^{-}=p_{c}-p^{+}$corresponds to the noise if the control is on.

The noise attenuation obtained with the implementation of nonlocal controls in the protected domain is discussed in the next section. A comparison between the noise attenuation obtained with application of local and nonlocal controls is also given there. Finally, the effect of some factors such as the Hausdorff distance $d_{H}$ between the external surface $\Gamma_{0}$ and boundary surface $\Gamma$, the number of sensors $N_{s}$ and controls $N_{c}$ are studied. 


\subsection{ASC with the implementation of nonlocal controls}

First, consider the extreme case of a numerous number of controls to reach the limit of the continuous control. Next, nonlocal controls, as many as 2304, are evenly distributed on each side of boundary surface $\Gamma$. Thereby, 13824 nonlocal controls are implied in total on the perimeter. The same quantity of sensors are supposed to be distributed on the external surface $\Gamma_{0}$ to detect the total field. Consider the frequency equal to $200 \mathrm{~Hz}$. The Hausdorff distance $d_{H}$ between the external surface $\Gamma_{0}$ and boundary surface $\Gamma$ is $0.2 \mathrm{~m}$ that means $d_{H} / \lambda=0.1$.

In case the desired sound source is not present, the sound field is shown in Figure 3a. With the implementation of the nonlocal control, the sound pressure is efficiently attenuated inside the cubic domain shielded. Figure 3b indicates the pressure contour lines in the protected domain. As shown, the noise is equal to $600 \mathrm{pa}(149 \mathrm{~dB})$ outside while the sound pressure is close to $40 \mathrm{pa}$ (126 dB) inside the shielded domain. This means that almost complete noise attenuation is achieved.

For the case of the desired sound present, the sound field with ASC is shown in Figures 3c. Figure 3d shows the sound pressure along interval $(-2.5,2.5)$ if $y=z=0$. As can be seen, with the ASC the total pressure retains the same outside the shielded domain while it coincides with the desired sound pressure inside the shielded domain. In other words, the noise is almost completely attenuated inside the shielded domain while the desired sound is preserved. More numerical simulations are further considered to analyze the properties of the algorithm. 


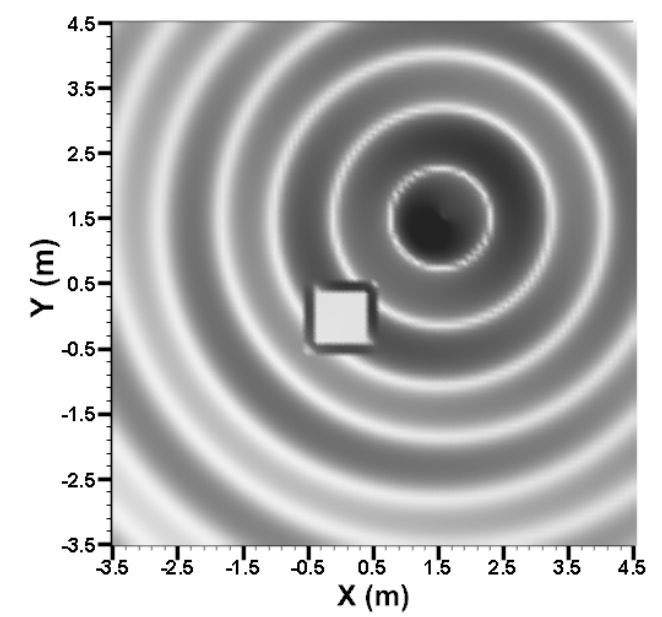

(a) Sound field (without desired sound)

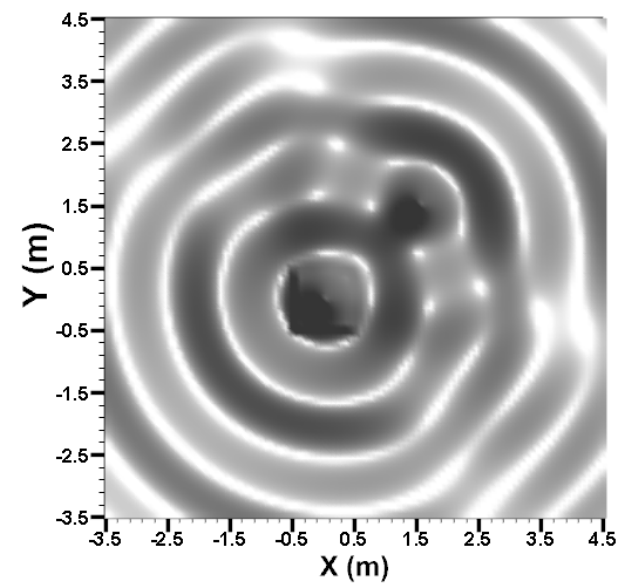

(c) Sound field (with desired sound)

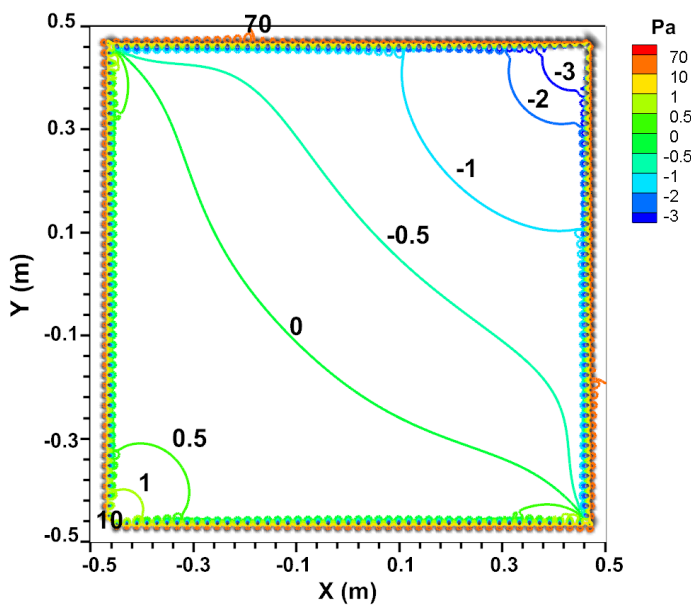

(b) Pressure contour lines

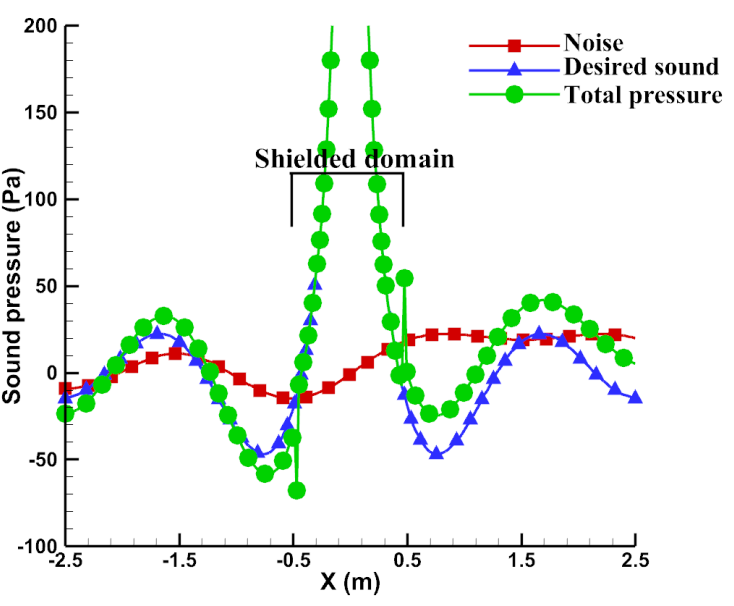

(d) Sound pressure with ASC

Fig. 3. Nonlocal potential-based ASC

\subsection{Comparison of noise attenuation achieved with local and nonlocal controls}

The noise attenuation achieved with the local and nonlocal controls are compared for frequency up to $200 \mathrm{~Hz}$. As noted above, in contrast to the local control, the operation of each nonlocal control is based on the total field over the entire boundary surface. First, assume that the total primary field is available for the control. Figure 4 demonstrates the distinction between the local control implied with and without existence of a desired sound source. In this test, 3 controls and 6 sensors are implied along each axis, respectively. The 
Hausdorff distance $d_{H}$ between the surfaces is equal to $0.2 \mathrm{~m}$. The minimal ratio between $d_{H}$ and the wave length $\lambda$ considered is equal to 0.1 . The noise attenuation is obtained with a mesh consisting of 100 observation points evenly spaced inside the cubic shielded domain.

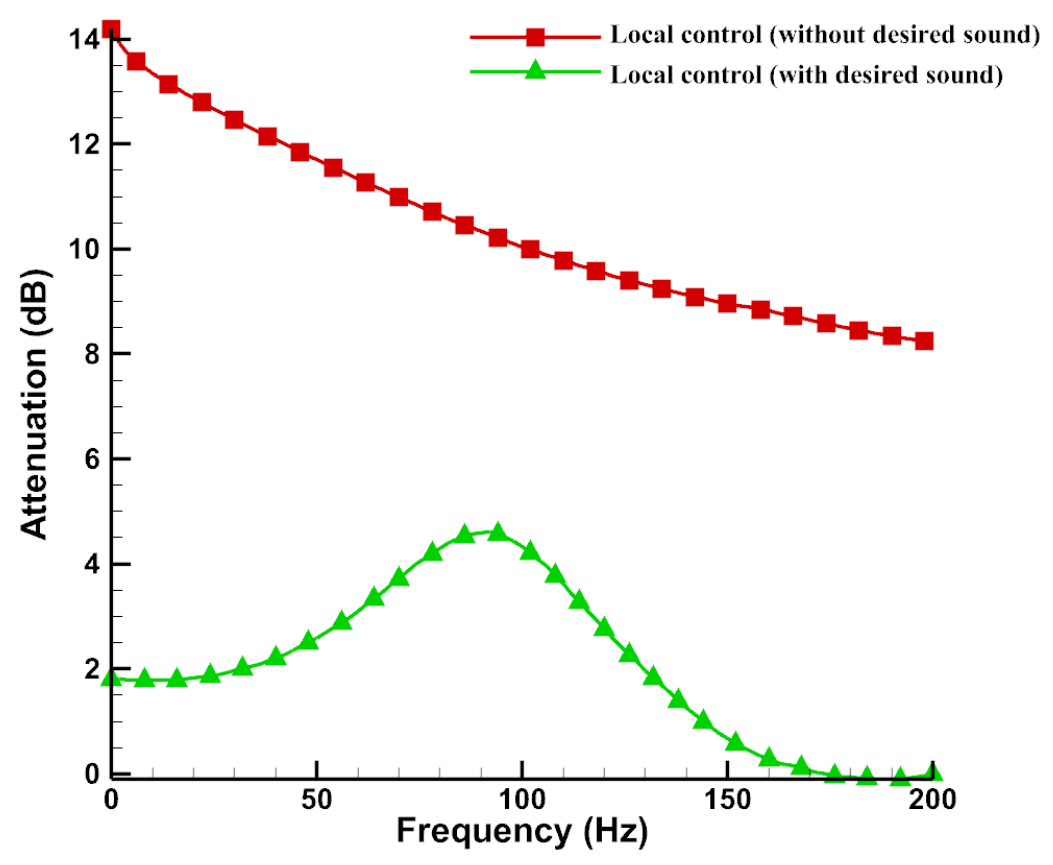

Fig. 4. Noise attenuation achieved by local controls with primary field available

As shown in Figure 4, a good noise attenuation is achieved with local controls if the desired source is not present. The average noise attenuation decreases monotonically with the increase of the frequency. Next, consider the case when desired sound is present. Even though the primary field is supposed to be known, the noise attenuation is quite poor and varies nonmonotonically with the growth of the frequency. It turns out that there is an effect of desired field on local controls. The explanation of this effect is as follows. As mentioned above, local controls depend on the total field at the vicinity of the boundary surface. The error in the calculation of the potentials with density $p_{\Gamma}^{+}$can be interpreted as noise. In other words, the control generates own noise apart form the noise attenuation. Thus, the presence of the desired sound results in 
deteriorations of the noise attenuation.

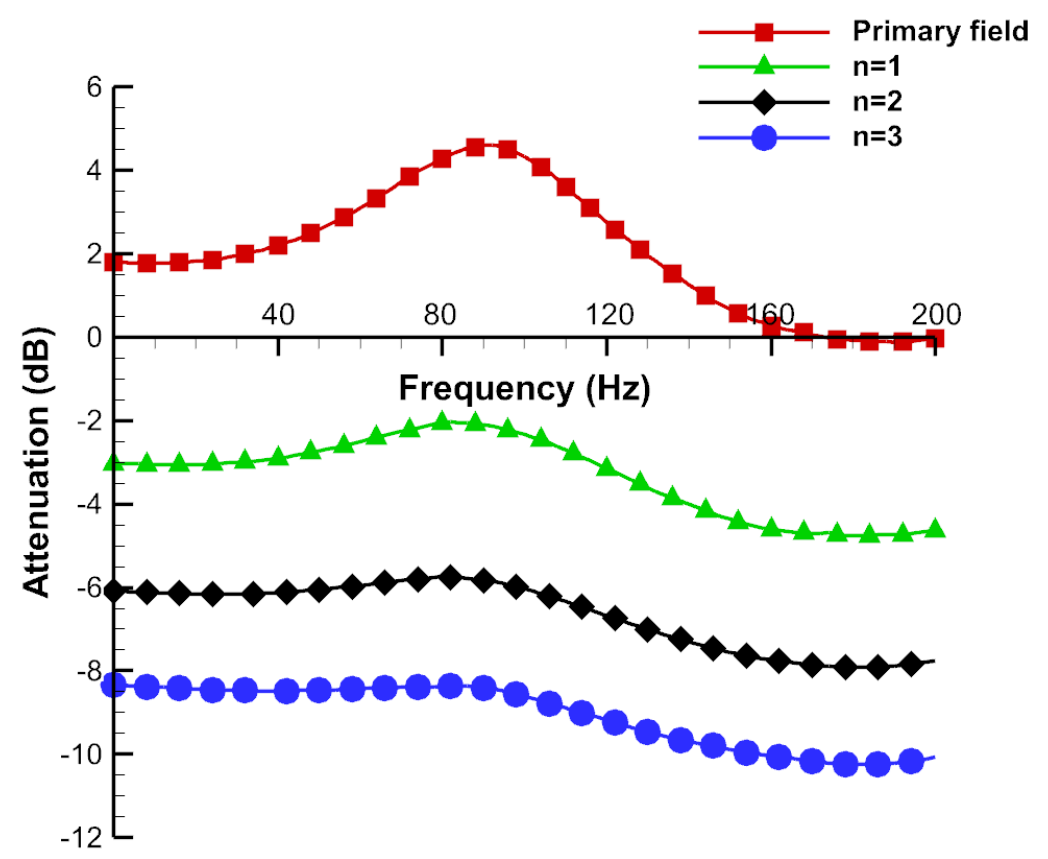

Fig. 5. Noise attenuation with local controls over pseudo-time steps $n$

In fact, the primary field is usually not available because the input data for the controls are practically affected by the secondary field. Next, consider how the control affects the input data if a desired sound exists. For that we analyze consequent pseudo-time steps. Under a pseudo-time step we understand a loop: input data - control - input data updated. Figure 5 shows the noise attenuation obtained by local controls after each pseudo-time step. The noise attenuation based on only the primary field coincides with that shown in the previous figure. Figure 5 demonstrates that the noise attenuation deteriorates with the number of pseudo-time steps from 1 to 3 . The noise attenuation even falls to negative values that means the noise is reinforced rather than attenuated inside the shielded domain. This phenomenon is caused by the reverse effect of controls.

The explanation of the reverse effect is the following. In the first pseudo time 
step, the continuous local control would generate an anti-noise equal to $-p^{-}$ inside the protected domain and a field equal to $p^{+}$outside [18]. Thus, it doubles the desired sound outside the shielded domain. This field is added to the input data for the second pseudo-time step. Then, the local control generates a field equal to $p^{+}$again and triple the contribution of desired sound outside the shielded domain at the second pseudo-time step, etc. On the one hand, the exact projection applied to $n p_{\Gamma}^{+}$is equal to zero. On the other hand, a discrete realization of the projection leads to extra noise which is proportional to the input field.

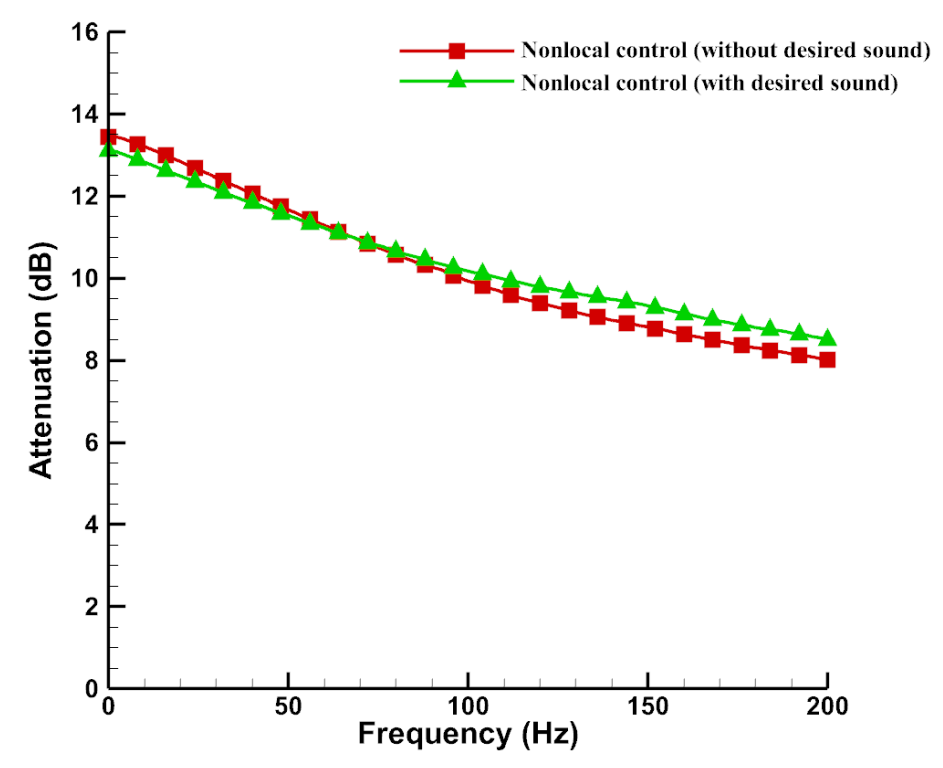

Fig. 6. Noise attenuation achieved by nonlocal controls

Next, we consider the noise attenuation obtained with nonlocal controls. Figure 6 shows that a good enough noise attenuation is achieved with nonlocal controls if the desired sound is not present. The noise attenuation obtained with nonlocal controls is similar to that obtained with local controls.

As can be seen from Figure 6, even if the desired sound is present, the noise attenuation with nonlocal control is practically the same as that without the 
desired sound. The noise attenuation drops monotonically with the growth of frequency in contrast to the local control based on the primary field. This result occurs because the contribution of desired sound as well as the noise caused by the discrete control is removed from the acoustic filed via the calculation of surface potentials on $\Gamma_{0}$ where the density of sensors essentially exceeds the density of controls on $\Gamma$.

Figure 7 demonstrates the noise attenuation obtained with nonlocal controls over pseudo-time steps. The input data is updated on external surface $\Gamma_{0}$ rather than boundary surface $\Gamma$. Figure 7 shows that the noise attenuation retains the same with the increase of the number of pseudo time steps.

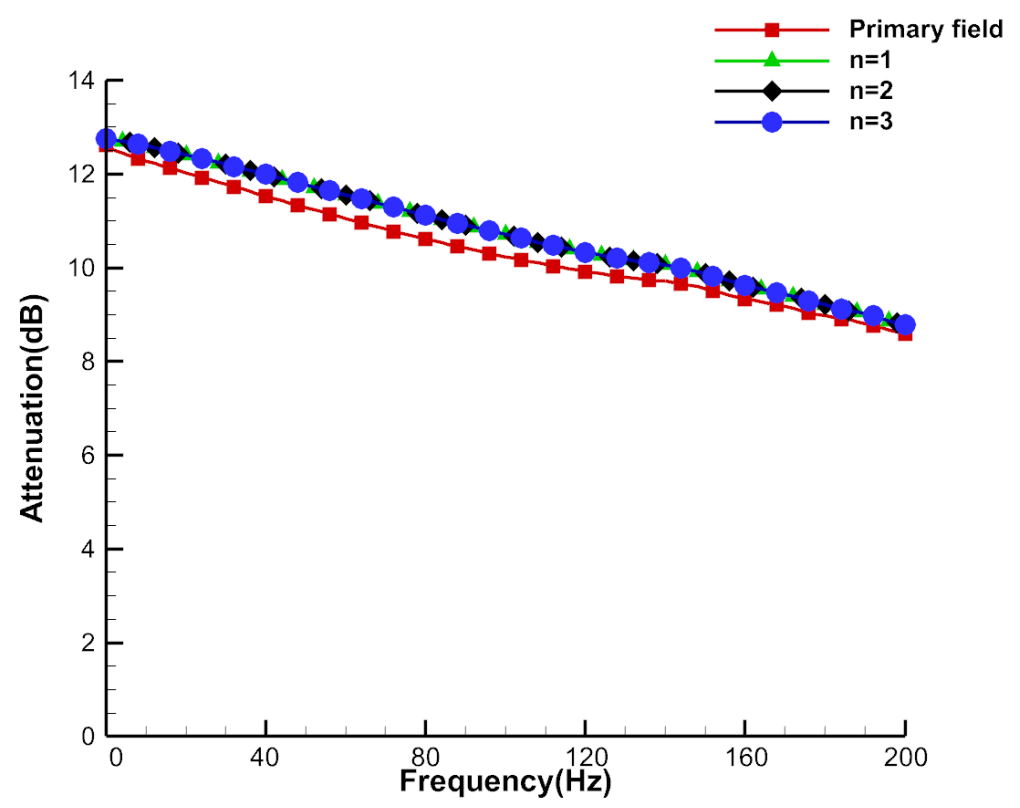

Fig. 7. Noise attenuation obtained by nonlocal controls over pseudo-time steps

\subsection{Sensitivity analysis}

The number of sensors and controls as well as the Hausdorff distance $d_{H}$ affect the level of noise attenuation. Next, we carry out some numerical experiments 
to analyze the effect of these factors on the noise attenuation.

First, a sensitivity analysis is considered with respect to the density of sensors. In this test, the number of controls along each axis $N_{c s}$ is equal to 3 . Thus, there are 9 controls on each side of boundary surface $\Gamma$ and 54 controls in total on the perimeter. In turn, sensors are uniformly distributed on external surface $\Gamma_{0}$ such that the number of sensors along each axis $N_{s s}=3,4,5,6$.

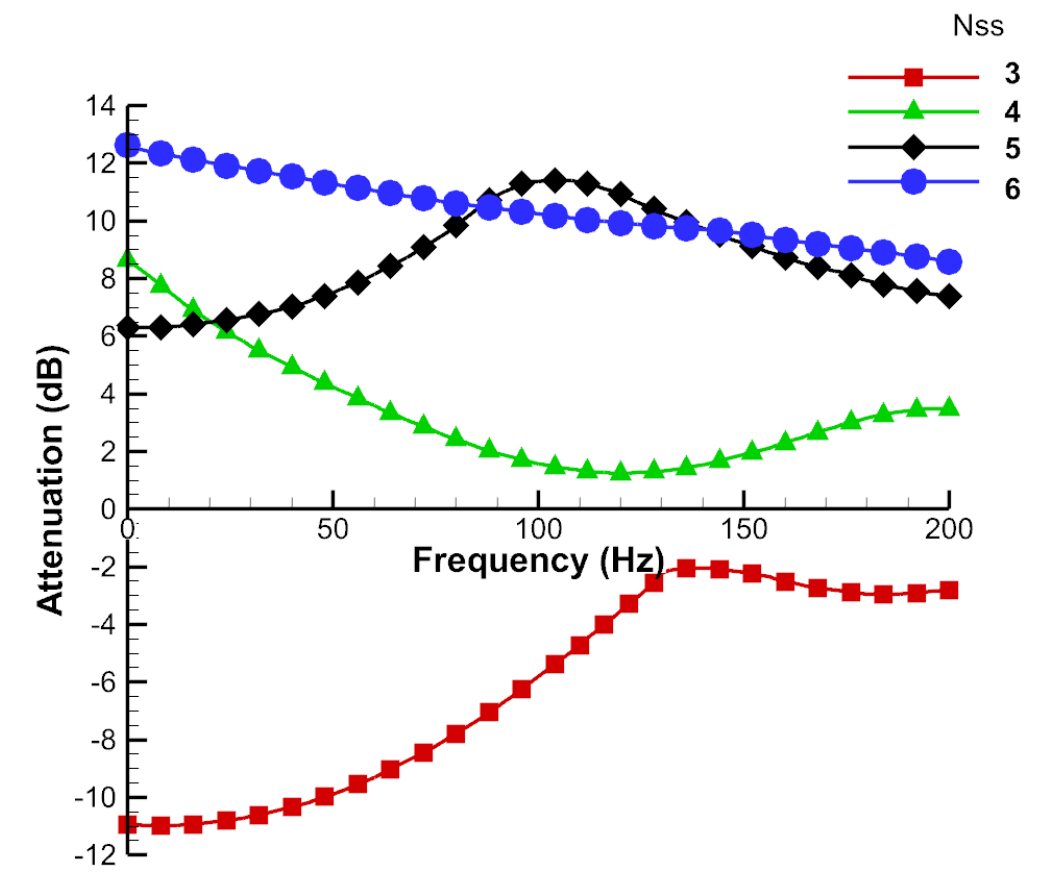

Fig. 8. Effect of the number of sensors $N_{s s}$ on noise attenuation

As shown in Figure 8, the noise attenuation obtained with 3 sensors along each axis is poor. A good enough noise attenuation is not achieved either if $N_{s s}$ is equal to 4 and 5 . In these cases the noise attenuation varies nonmonotonically with the increase of frequency. The reason of this is similar to that for the local control with a desired sound. In turn, a reasonably good noise attenuation, over $10 \mathrm{~dB}$, is achieved with $N_{s s}$ equal to 6 . Therefore, the ratio of $N_{s s}$ and $N_{c s}$ is recommended to be above 2 in this case. The low ratio of $N_{s s}$ and $N_{c s}$ leads an error in the calculation of surface potentials due to the discrete sets 
of sensors. Figure 9 demonstrates the minimal ratio of $N_{s s}$ and $N_{c s}$ needed to achieve the noise attenuation close to that obtained with the local control if the primary field $p_{\Gamma}$ is known. With the growth of $N_{c s}$, less ratio of $N_{s s}$ and $N_{c s}$ close to 1.5 , is required for the nonlocal control.

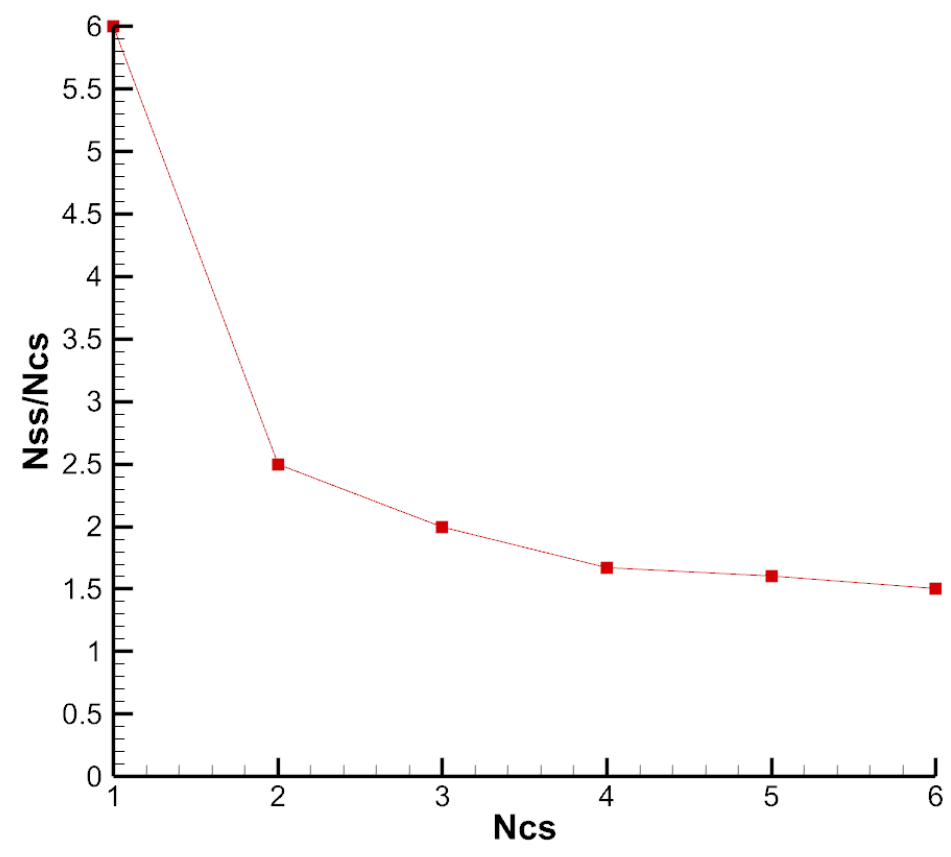

Fig. 9. The minimal ratio of $N_{s s}$ and $N_{c s}$ required for the nonlocal control

Next, consider the effect of the number of controls on the ASC. In this case, the sound frequency and $N_{s s}$ are equal to $200 \mathrm{~Hz}$ and 8, respectively. Several sets of controls $\left(N_{c s}=2,3,4,5\right)$ are implemented. First, Figure 10 represents the sound pressure in domain $D^{-}$and the pressure contour lines inside the shielded domain $D^{+}$. As shown in this figure, the absolute sound pressure is close to $4 \mathrm{pa}(106 \mathrm{~dB})$ at the center of the shielded domain if $N_{c s}=4$ (see Figures 10a and 10b). The absolute sound pressure increases from $4 p a$ to 8 pa $(112 \mathrm{~dB})$ at the center of the protected domain if $N_{c s}$ decreases from 4 to 2 (see Figures 10c and 10d). In addition, it is worth noting that the controls become more individually distinguishable if $N_{c s}$ is reduced from 4 to 2 . Local peaks around each secondary source occur. 


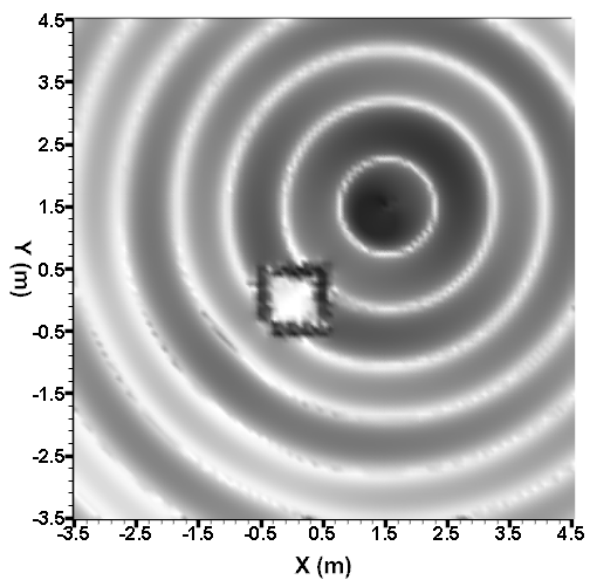

(a) $N_{c s}=4$. Sound pressure

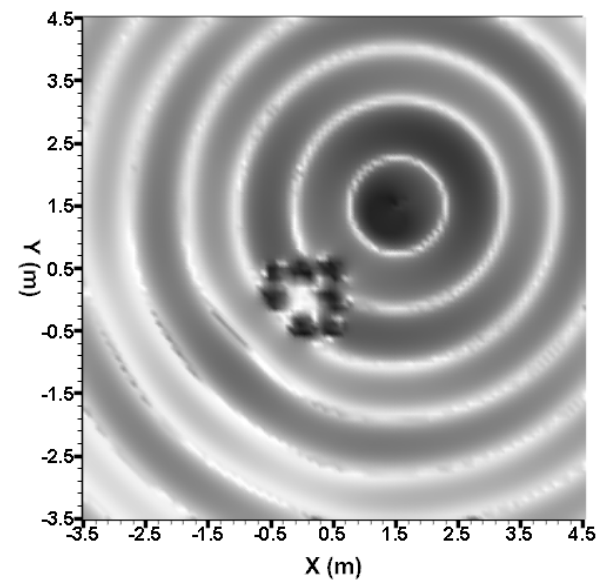

(c) $N_{c s}=2$. Sound pressure

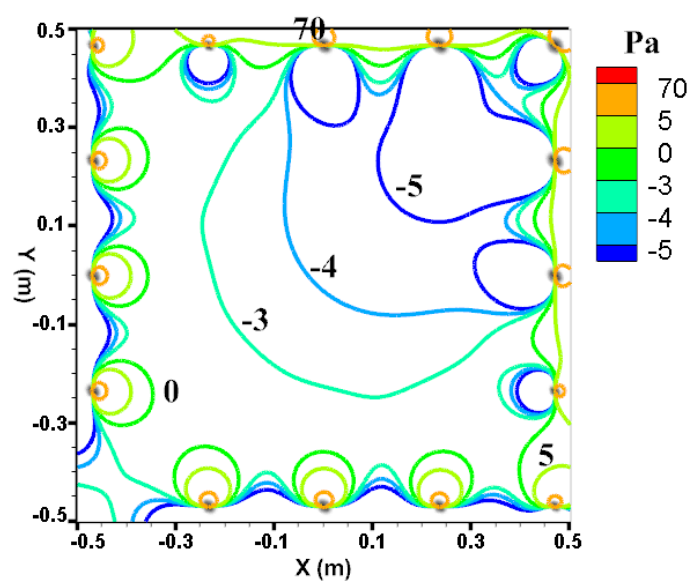

(b) $N_{c s}=4$. Pressure contour line

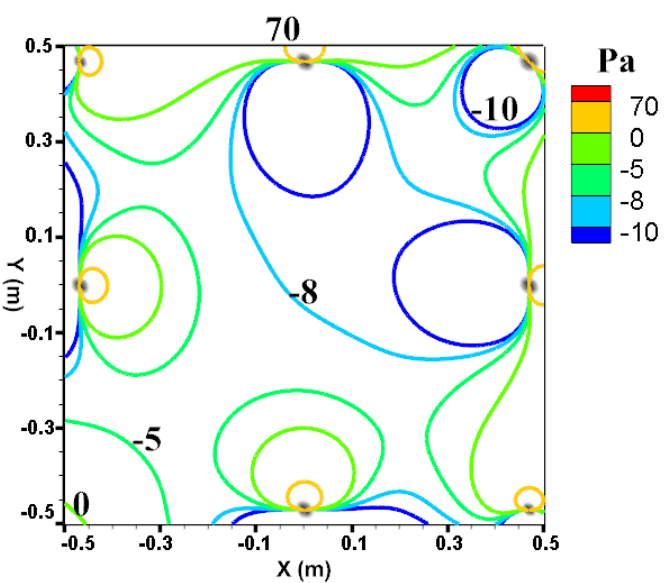

(d) $N_{c s}=2$. Pressure contour line

Fig. 10. Sound pressure with ASC $\left(N_{c s}=2,4\right)$

Then, presume that the total primary field is available for the control. Figures $11 \mathrm{a}$ and $11 \mathrm{~b}$ demonstrate the noise attenuation obtained with several sets of local controls with and without the presence of desired sound respectively. In both cases the noise attenuation grows with the increase of the number of controls. However, due to the contribution of desired sound, the noise attenuation achieved by local controls without the desired sound is much better than that obtained with the preservation of desired sound. The noise attenuation obtained with and without the presence of desired sound are equal to $8 d B$ and $18 \mathrm{~dB}$ respectively at $40 \mathrm{~Hz}$ if $N_{c s}$ is equal to 5 . It is worth noting that there 


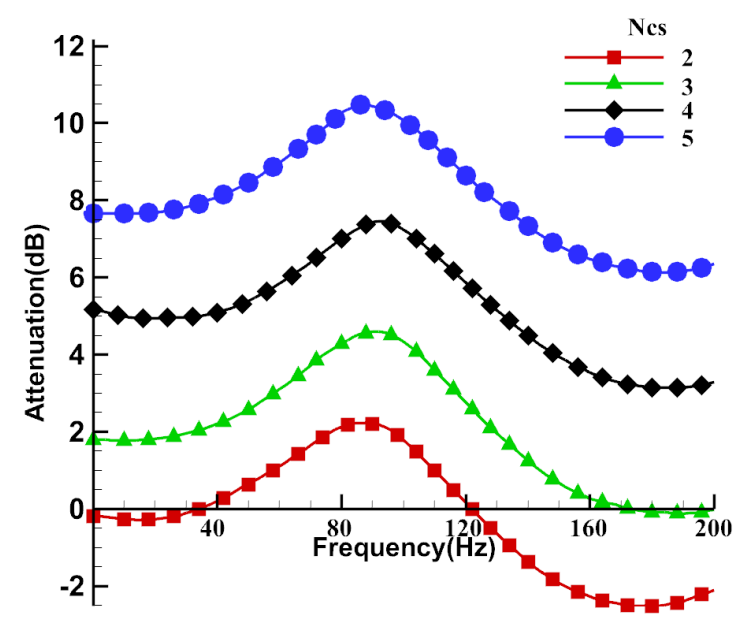

(a) Local controls (with $p^{+}$)

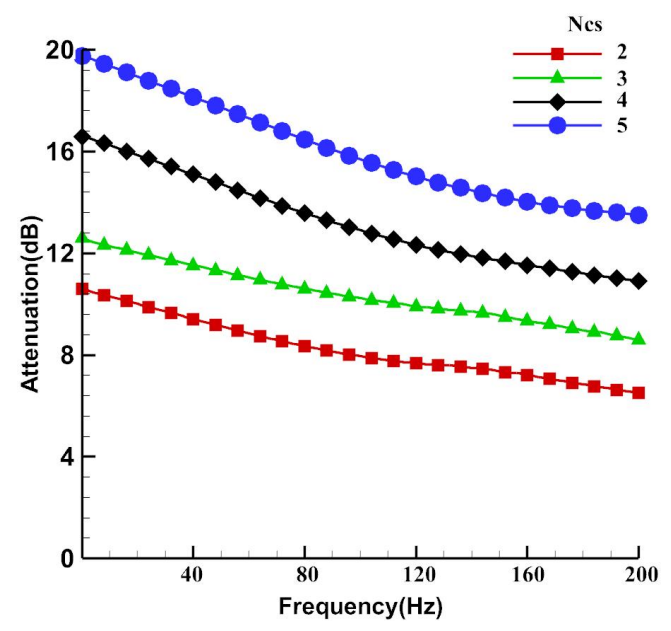

(c) Nonlocal controls (with $p^{+}$)

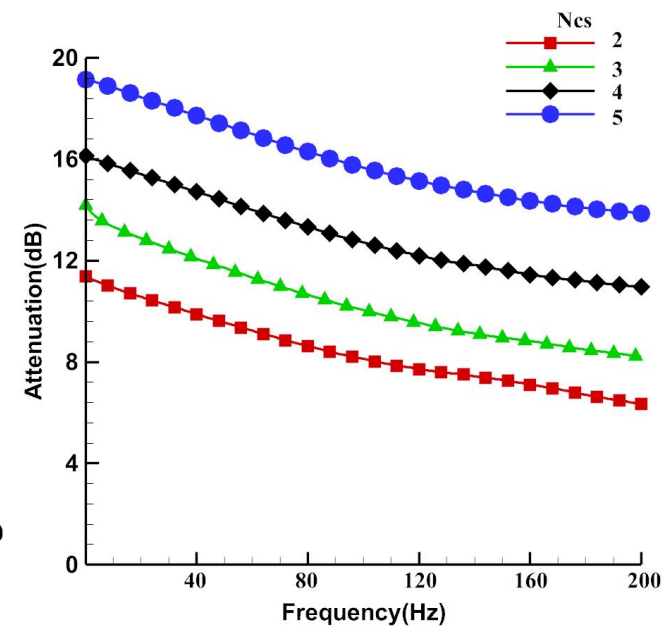

(b) Local controls $\left(p^{+}=0\right)$

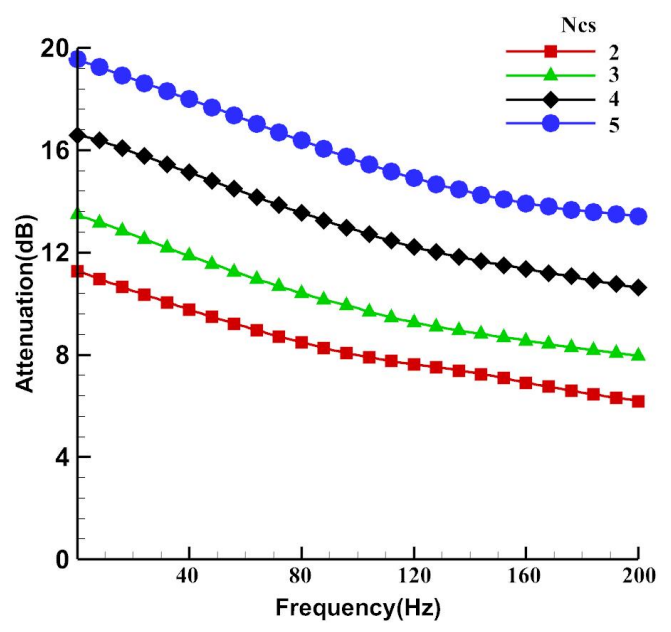

(d) Nonlocal controls $\left(p^{+}=0\right)$

Fig. 11. Effect of the number of controls $N_{c s}$ on noise attenuation with the input data based on the primary field

is a negative noise attenuation if $N_{c s}$ is reduced to 2 while the desired sound is present. This means the noise is even amplified rather than attenuated by ASC in this case. Thus, the effect of desired sound on controls becomes more obvious with the decrease of the number of controls $N_{c s}$. This is an expected result since the deterioration in the noise attenuation is related to a discrete realization of the projection.

Figures $11 \mathrm{c}$ and $11 \mathrm{~d}$ demonstrate the noise attenuation achieved with nonlocal 
controls with and without the presence of desired sound source. As can be seen, a good enough level of noise attenuation can be retained while the desired sound is present.

Finally, we consider the effect of the Hausdorff distance $d_{H}$ between surfaces $\Gamma$ and $\Gamma_{0}$ on the noise attenuation obtained with nonlocal controls. Figure 12 demonstrates the relationship between the Hausdorff distance $d_{H}$ and the noise attenuation. In this test, the number of controls $N_{c s}$ and sensors $N_{s s}$ is assumed to be 3 and 6 , respectively. The results show that a significant noise attenuation is achieved if $d_{H}$ is greater than $0.2 \mathrm{~m}$. However, the achieved noise attenuation is not satisfactory if the Hausdorff distance $d_{H}$ is equal to $0.15 \mathrm{~m}$. Thus, the minimal ratio between $d_{H}$ and the wave length $\lambda$ to be attenuated is equal to 0.06 . For example, a negative noise attenuation of about $-5 d B$ is achieved at $50 \mathrm{~Hz}$ if $d_{H}$ decreases to $0.1 \mathrm{~m}$. This is because the nonlocal controls operate locally if the external surface $\Gamma_{0}$ is too close to the boundary $\Gamma$. As can be seen in Figure 10, there is a clear local effect of each control source. 


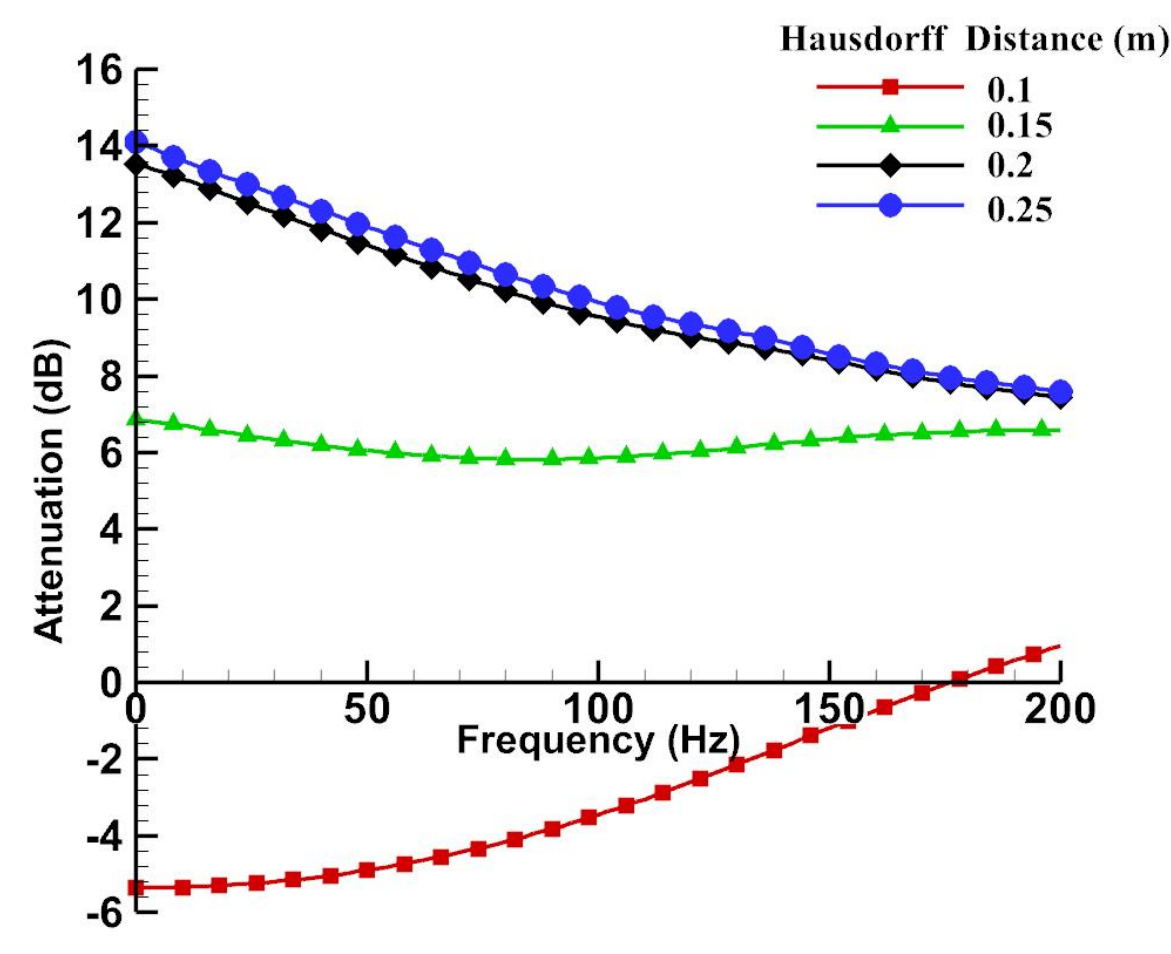

Fig. 12. Effect of Hausdorff distance $d_{H}$ on noise attenuation

\subsection{A line noise source}

Consider now the case of undesired sound generated by a finite length line source. It is supposed to be situated parallel to axis $y$ with the centre at point with coordinate $(30 \mathrm{~m}, 30 \mathrm{~m}, 0 \mathrm{~m})$. The length of the line source $l$ is equal to $3 \mathrm{~m}$. The desired sound source is identical to that considered at the previous test cases. The number of controls $N_{c s}$ and the Hausdorff distance $d_{H}$ between surfaces are equal to 4 and $0.2 \mathrm{~m}$ respectively. The frequency is equal to 100 $H z$. Based on the sensitivity analysis, the minimal ratio of $N_{s s}$ and $N_{c s}$ is 1.75 while $N_{c s}$ is equal to 4 . Thus, 7 sensors are uniformly implemented along each axis.

Under far-field conditions the sound pressure generated by a finite length line source is given by [31]: 


$$
p(x, y, z)=\frac{\sin \left(\frac{k l}{2} \sin \theta\right)}{\frac{k l}{2} \sin \theta} \frac{j w \rho Q e^{-j k r}}{4 \pi r}
$$

where $w$ is the angular frequency of the wave; $\rho$, the density of the medium; $Q$, the volume velocity; $r$, the distance to the source from a receiver, and $\theta$ is the angle between the normal to the source $(1,0,0)$ and direction to the receiver.

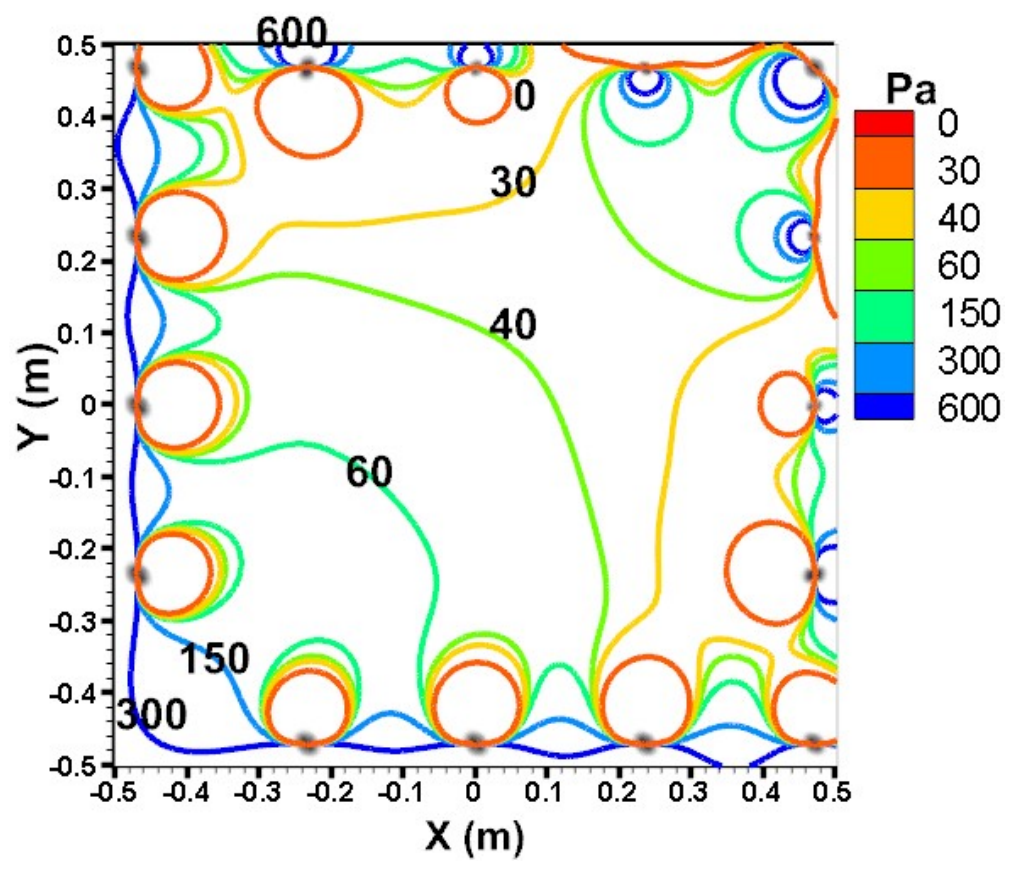

Fig. 13. Pressure contour lines (finite length line source)

The sound pressure generated by the finite length line source is attenuated with the nonlocal ASC. In case the desired sound source is not present, the pressure contour lines inside the protected domain is shown in Figure 13. As shown, the noise is equal to $600 \mathrm{pa}(149 \mathrm{~dB})$ outside the shielded domain while the sound pressure is close to $40 \mathrm{pa}(126 \mathrm{~dB})$ at the center of the cube.

Next, we consider the noise attenuation obtained with the growth of frequency with and without desired sound. As can be seen from Figure 14a, in case the 
desired sound is present, the noise attenuation with nonlocal control is practically the same as that without the desired sound. Figure 14b demonstrates the noise attenuation obtained with nonlocal controls over pseudo-time steps. Figure 14b shows that the noise attenuation retains almost the same with the increase of the number of pseudo time steps. Thus, as can be expected, practically the same conclusions obtained for a point noise source are applicable to an an extended source.

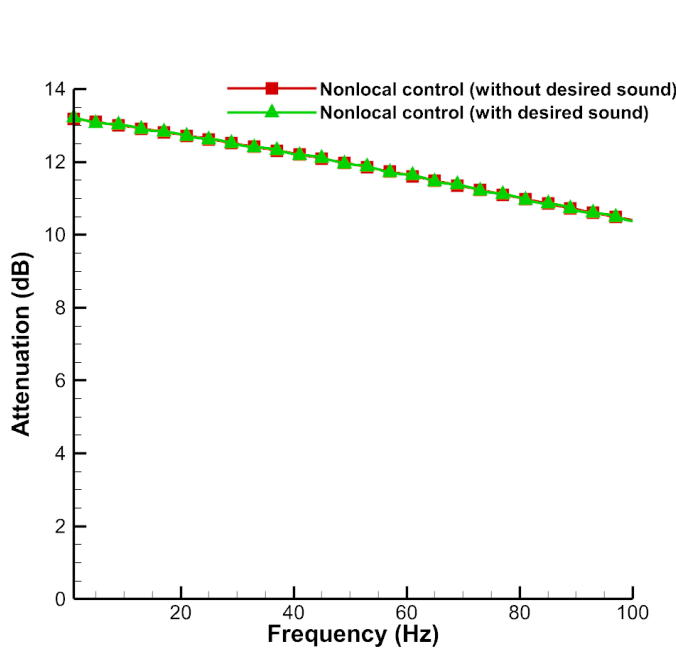

(a) Noise attenuation

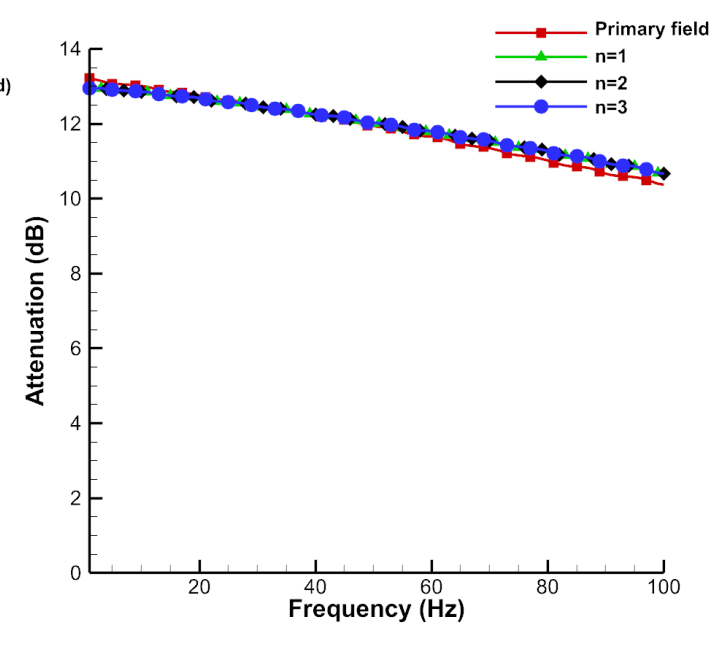

(b) Noise attenuation over pseudo-time steps

Fig. 14. Nonlocal potential-based ASC (finite length line source)

\section{Conclusion}

It has been shown that the nonlocal potential-based method of ASC can efficiently attenuate noise while preserving desired sound. The input data for the controls correspond to the real-time regime when both primary and secondary sources make the contribution. It has been demonstrated for the first time that even if the total primary field is known at the perimeter of the domain to be shielded, a poor noise attenuation can be achieved by local controls in case 
when the desired sound is present and a small number of controls operate. Moreover, a steady noise attenuation becomes impossible with the local control if the reverse effect of control on the input data is to be counted. As has been demonstrated, the nonlocal potential-based ASC is capable of attenuating incoming noise with the presence of a desired sound component even with a relatively small number of sensors and controls. It is demonstrated that in the general case the density of sensors is more important for the noise attenuation than the density of the controls. In addition, it has been shown that the surface of the sensors should not be situated too close to the surface with the controls. In the future work, the location of sensors and actuators should be optimized not only to increase the level of noise attenuation but also to improve the controllability and observability of the system.

\section{Acknowledgement}

The authors are grateful to unknown referees for useful remarks which help improve the quality of the paper.

\section{References}

[1] J. F. Williams, Review lecture-anti-sound, Proc. R. Soc. Lond. A 395 (1808) (1984) 63-88.

[2] G. Malyuzhinets, An unsteady diffraction problem for the wave equation with compactly supported right-hand side, Proceedings of the Acoustics Institute, USSR Academy of Sciences (1971) 124-139. 
[3] V. S. Ryaben'kii, Method of difference potentials and its applications, Vol. 30, Springer Science \& Business Media, 2012.

[4] J. Loncaric, V. Ryaben'kii, S. Tsynkov, Active shielding and control of noise, SIAM J Applied Mathematics 62 (2) (2001) 563-596.

[5] J. Loncaric, S. V. Tsynkov, Optimization of acoustic source strength in the problems of active noise control, SIAM J Applied Mathematics 63 (4) (2003) 1141-1183.

[6] V. Ryaben'kii, S. Utyuzhnikov, A. Turan, On the application of difference potential theory to active noise control, Advances in Applied Mathematics 40 (2) (2008) 194-211.

[7] V. Ryaben'kii, S. Tsynkov, S. Utyuzhnikov, Inverse source problem and active shielding for composite domains, Applied Mathematics Letters 20 (5) (2007) 511-515.

[8] A. Peterson, S. Tsynkov, Active control of sound for composite regions, SIAM J Applied Mathematics 67 (6) (2007) 1582-1609.

[9] V. Ryaben'kii, S. Tsynkov, S. Utyuzhnikov, Active control of sound with variable degree of cancellation, Applied Mathematics Letters 22 (12) (2009) 1846-1851.

[10] S. V. Utyuzhnikov, Nonlinear problem of active sound control, J Computational and Applied Mathematics 234 (1) (2010) 215-223.

[11] S. V. Utyuzhnikov, Non-stationary problem of active sound control in bounded domains, J Computational and Applied Mathematics 234 (6) (2010) 1725-1731.

[12] H. Lim, S. Utyuzhnikov, Y. Lam, A. Turan, M. Avis, V. Ryaben'Kii, S. Tsynkov, Experimental validation of the active noise control methodology based on difference potentials, AIAA J 47 (4) (2009) 874-884.

[13] H. Lim, S. Utyuzhnikov, Y. Lam, A. Turan, Multi-domain active sound 
control and noise shielding, J Acoustical Society of America 129 (2) (2011) $717-725$.

[14] H. Lim, S. Utyuzhnikov, Y. Lam, L. Kelly, Potential-based methodology for active sound control in three dimensional settings, J Acoustical Society of America 136 (3) (2014) 1101-1111.

[15] E. A. Ntumy, S. V. Utyuzhnikov, Active sound control in composite regions, Applied Numerical Mathematics 93 (2015) 242-253.

[16] E. A. Ntumy, S. V. Utyuzhnikov, Active sound control in 3D bounded regions, Wave Motion 51 (2) (2014) 284-295.

[17] M. Fedoryuk, An unsteady problem of active noise suppression, Acoustic J 22 (1976) 439-443.

[18] V. S. Ryaben'kii, S. V. Utyuzhnikov, Active shielding model for hyperbolic equations, IMA J Applied Mathematics 71 (6) (2006) 924-939.

[19] N. Epain, E. Friot, Active control of sound inside a sphere via control of the acoustic pressure at the boundary surface, J Sound and Vibration 299 (3) (2007) 587-604.

[20] C. D. Petersen, R. Fraanje, B. S. Cazzolato, A. C. Zander, C. H. Hansen, A kalman filter approach to virtual sensing for active noise control, Mechanical Systems and Signal Processing 22 (2) (2008) 490-508.

[21] B. Kwon, Y. Park, Active window based on the prediction of interior sound field: experiment for a band-limited white noise, in: INTER-NOISE and NOISE-CON Congress and Conference Proceedings, Vol. 2011, Institute of Noise Control Engineering, 2011, pp. 443-446.

[22] A. Jakob, M. Möser, Active control of double-glazed windowspart i: Feedforward control, Applied Acoustics 64 (2) (2003) 163-182.

[23] Jakob, Andre and Möser, Michael, Active control of double-glazed windows. part ii: Feedback control, Applied Acoustics 64 (2) (2003) 183-196. 
[24] H. Huang, X. Qiu, J. Kang, Active noise attenuation in ventilation windows, J Acoustical Society of America 130 (1) (2011) 176-188.

[25] S. V. Utyuzhnikov, A practical algorithm for real-time active sound control with preservation of interior sound, Computers \& Fluids 157 (2017) 175-181.

[26] S. V. Utyuzhnikov, Generalized Calderón-Ryaben'kii's potentials, IMA J Applied Mathematics 74 (1) (2009) 128-148.

[27] S. V. Utyuzhnikov, Real-time active wave control with preservation of wanted field, IMA J Applied Mathematics 79 (6) (2014) 1126-1138.

[28] V. Mallardo, M. Aliabadi, A. Brancati, V. Marant, An accelerated BEM for simulation of noise control in the aircraft cabin, Aerospace Science and Technology 23 (1) (2012) 418-428.

[29] A. Brancati, M. Aliabadi, Boundary element simulations for local active noise control using an extended volume, Engineering analysis with boundary elements 36 (2) (2012) 190-202.

[30] A. Brancati, M. Aliabadi, V. Mallardo, A BEM sensitivity formulation for three-dimensional active noise control, International Journal for $\mathrm{Nu}$ merical Methods in Engineering 90 (9) (2012) 1183-1206.

[31] L. E. Kinsler, A. R. Frey, A. B. Coppens, J. V. Sanders, Fundamentals of acoustics, Fundamentals of Acoustics, 4th Edition, by Lawrence E. Kinsler, Austin R. Frey, Alan B. Coppens, James V. Sanders, pp. 560. ISBN 0-471-84789-5. Wiley-VCH, December 1999. (1999) 560. 